\title{
Búsqueda de resistencia a amantadina en cepas de virus influenza $A$ aisladas en Santiago de Chile, entre los años 2001 y 2002
}

\author{
ELISA FEHLMANN P., NICOLE LE CORRE P., \\ KATIA ABARCA V., PAULA GODOY M., LUISA MONTECINOS P., \\ ANA VELOZ B. y MARCELA FERRÉS G.
}

\section{Search of amantadine-resistance in influenza A strains isolated in Santiago, Chile, 2001-2002}

Amantadine has been used for prevention and treatment of influenza A infection. It blocks the proton through the M2 ion channel. Drug-resistant viruses appear quickly when this therapy is used. Single amino acids changes in the $\mathrm{H}_{2}$ protein can confer resistance, being the most frequent one in position 31. Different methods to detect resistant strains have been described. The objectives were to determine the existence of amantadine resistance of influenza A strains isolated in a virologic laboratory in Santiago, Chile, between 2001-2002, and to validate a new molecular method to detect resistant strains. A PCR restriction fragment length polymorphism analysis was employed for the detection of resistant viruses. In 31 processed strains no mutation in the position 31 was found. This result supports that amantadine resistance is very low or absent in Chile. This could be explained by a limited use of this drug in the study population. This method could be used as a monitoring system to survey resistant viruses.

Key words: Amantadine; Resistance; Influenza A virus; M2 protein.

Palabras claves: Amantadina; Resistencia; Virus influenza A; Proteína M2.

\section{Introducción}

El virus influenza (FLU) causante de la gripe $u$ otros síndromes respiratorios altos o bajos, se asocia a una significativa morbi-mortalidad, en especial en sujetos con ciertas condiciones de riesgo. Determina frecuente ausentismo escolar y laboral durante las epidemias, como también hospitalización por sus complicaciones, con un alto costo económico ${ }^{1}$. Si bien el uso de la vacuna ha permitido reducir la incidencia y complicaciones en grupos de riesgo, no ha logrado un control completo de la infección ${ }^{2}$. Esto es atribui- do a una eficacia protectora limitada (60-70\%), la necesidad de revacunaciones anuales, a coberturas parciales de las campañas de vacunación en la población general y a la elevada variabilidad antigénica del virus. La protección proporcionada por la vacuna varía entre 50 y $95 \%$ dependiendo además de la similitud entre las cepas incluidas en la vacuna y la cepa salvaje circulante ${ }^{3}$.

Por ello los antivirales tienen aún un rol importante en el manejo y prevención de la FLU. Uno de los antivirales más usados, licenciado en la década de los 60', es amantadina. Su mecanismo de acción es bloquear la replicación viral median-

\footnotetext{
Pontificia Universidad Católica de Chile, Santiago Chile:

Residente Servicio de Pediatría, Hospital Clínico (EFP, NLCP).

Departamento de Pediatría (KAV, MFG).

Laboratorio de Infectología y Virología Molecular (KAV, PGM, LMP, AVB, MFG).
}

Proyecto financiado por: Escuela de Medicina de la Pontificia Universidad Católica de Chile (Proyecto de investigación para becados, PG-30/02).

Recibido: 16 abril 2004

Aceptado: 1 febrero 2005 
te inhibición de la proteína M2, presente en la envoltura del virus, cuya función es facilitar su denudamiento dentro de la célula. La proteína M2 actúa como un canal iónico que permite la entrada de iones al virión endocitado, con la resultante fusión de las membrana viral y endosomal. De esta forma, el material genético viral es liberado dentro de la célula infectada permitiendo su posterior replicación al interior del núcleo ${ }^{1,4}$ (Figura 1). Dado que la proteína M2 está presente solamente en los virus FLU A, el uso de este antiviral no está indicado en casos de FLU B. Diversos estudios han mostrado que amantadina reduce la transmisión viral y acorta la duración de la fiebre en un día, así como también la severidad de los síntomas $^{5-7}$. De esta manera, este fármaco cumple un rol importante en el tratamiento contra la FLU A ${ }^{8}$.

La aparición de resistencia a amantadina en relación a su uso profiláctico y terapéutico ha generado una preocupación de salud pública. Este fenómeno se ha observado principalmente en comunidades cerradas como son los asilos de ancianos e instituciones de salud ${ }^{9-10}$. Respecto a la frecuencia de adquisición de esta resistencia, distintos estudios muestran resultados muy variables: desde 10 a $30 \%$ en algunas series ${ }^{6,9,11-13}$ a 1,5 y $3,4 \%$ en otras ${ }^{10-14}$. La generación de resistencia ocurre entre los 2 y 7 días de iniciado el tratamiento habiéndose documentado propagación de virus resistentes entre los contactos cer$\operatorname{canos}^{6,13}$. La adquisición de resistencia se debe a mutaciones en los genes que codifican para los aminoácidos constituyentes del dominio transmembrana de la proteína M2. Estas mutaciones ocurren en las posiciones $26,27,30$ y 31 , siendo esta última la más frecuente ya que da cuenta de 70 a $80 \%$ de los $\operatorname{casos}^{12}$.

La metodología tradicional para la detección de cepas resistentes en el laboratorio es compleja y consiste en demostrar la pérdida de la capacidad de inhibir cultivos celulares en presencia de amantadina ${ }^{15}$. Procedimientos más modernos incluyen amplificación mediante RPC, clonación y secuenciación del material genético que codifica a la proteína M2 en cepas de virus FLU expuestos a amantadina ${ }^{16,17}$. Otra metodología para determinar la presencia de estas mutaciones es el análisis de los tamaños de fragmentos que codifican para la proteína M2, amplificados por RPC y procesados con enzimas de restricción ${ }^{12,18}$.

En nuestro medio no se ha reportado la existencia de cepas de virus FLU A resistentes a amantadina. Si bien este medicamento es recomendado como terapia y profilaxis, no se conoce la frecuencia de uso en Chile. La hipótesis de los investigadores es que la frecuencia de resistencia a amantadina en las cepas de virus FLU A chilenas es muy baja o inexistente. Los objetivos de este estudio fueron estudiar la existencia de resistencia a amantadina de las cepas de virus FLU A aisladas entre los años 2001 y 2002 en un laboratorio de virología implementando técnicas de biología molecular para su detección.

\section{Material y Métodos}

Se utilizaron cepas de virus FLU A aisladas en el Laboratorio de Infectología y Virología Molecular de la Pontificia Universidad Católica de Chile. Estas cepas provenían de pacientes hospitalizados y ambulatorios, obtenidas mediante cultivo en líneas celulares de riñón de perro (MDCK) de muestras de hisopado nasofaríngeo empleando el procedimiento habitual en dicho laboratorio $^{19}$. El diagnóstico de FLU A fue realizado luego de 48 horas de cultivo mediante detección de antígenos específicos por inmunofluorescencia (Bartels, Viral Respiratory Screening and Identification Kit) siguiendo las instrucciones del fabricante. Posteriormente las cepas fueron almacenadas a $-70^{\circ} \mathrm{C}$ hasta su procesamiento.

Se trabajó con cepas aisladas y almacenadas durante los años 2001 y 2002. Se recolectó información demográfica y antecedentes de vacunación anti FLU y uso de antivirales en estos pacientes mediante revisión de las fichas clínicas y/o llamadas telefónicas.

Brevemente, la metodología consistió en amplificar mediante RPC una región del genoma que codifica para la proteína M2 de 150 pares de bases (pb) que contiene la posición 31 y posteriormente, diferenciar genomas con y sin mutación mediante digestión con enzimas de restricción. Específicamente, la enzima de restricción Sca I permite esta diferenciación por cuanto corta el fragmento de la cepa salvaje en dos fragmentos de 120 y $30 \mathrm{pb}$, mientras que no corta en presencia de la mutación, manteniendo el fragmento de $150 \mathrm{pb}^{12}$. La observación de los fragmentos resultantes post digestión en gel de agarosa permite la diferenciación de cepas con y sin mutación en la posición 31 (Figura 2).

Extracción del ARN viral. Luego de descongelar las cepas almacenadas se realizó extracción de ARN viral utilizando un kit de extracción comercial (High Pure ARN Isolation Kit, Roche), siguiendo las instrucciones del fabricante. Se procesaron de la misma forma 7 muestras de agua como controles del procedimiento. 


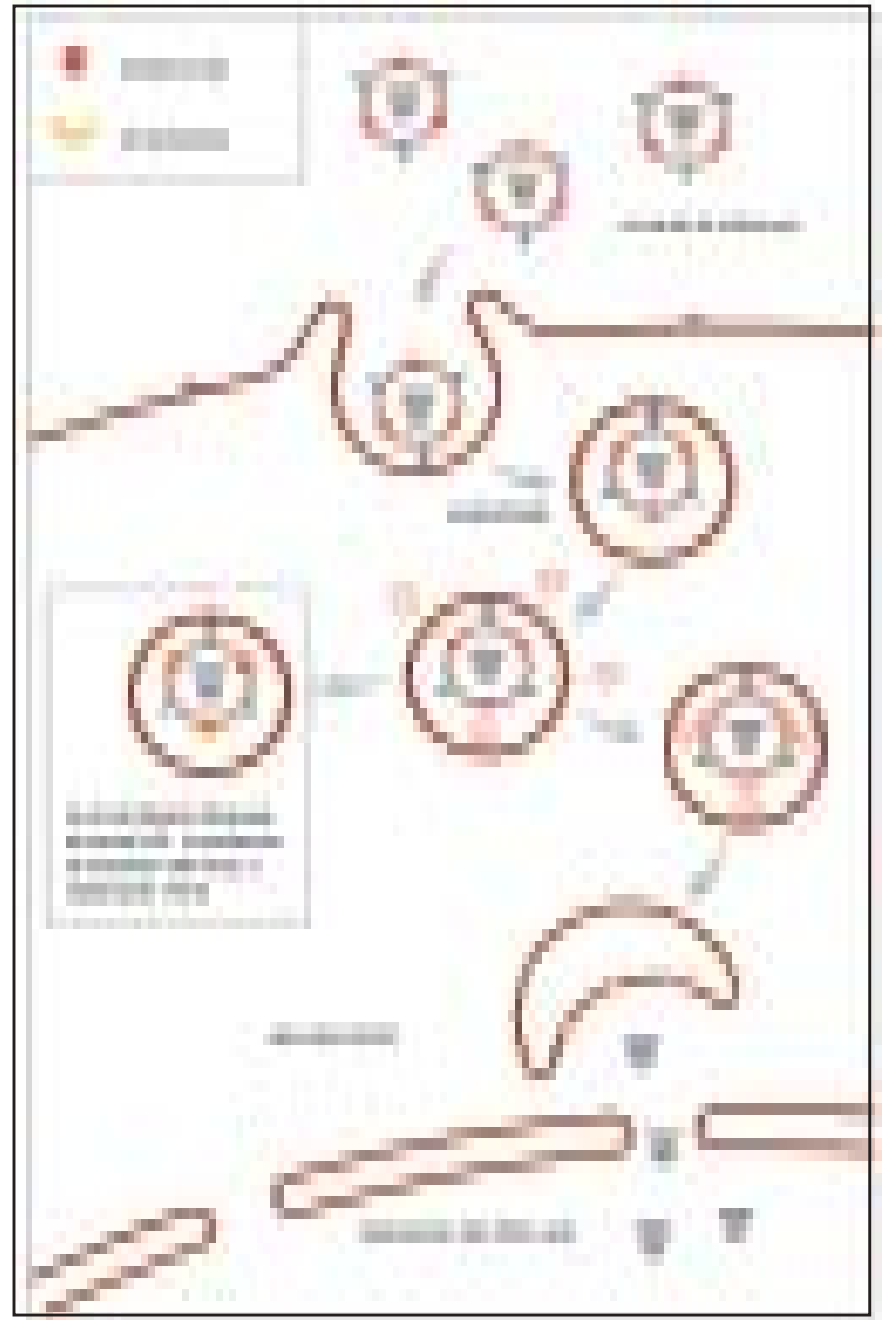

Figura 1. Función de la proteína M2 en la replicación viral. Rol de la amantadina como antiviral.

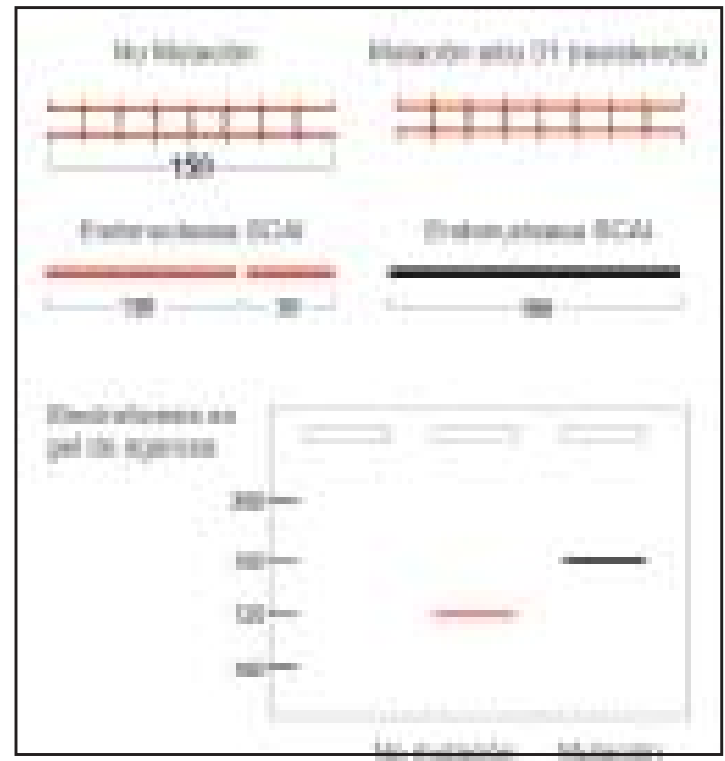

Figura 2. Esquema de la metodología utilizada para identificar la mutación 31: digestión con enzima de restricción Sca I.
Obtención de ADN complementario. Las hebras de ARN obtenidas fueron sometidas a transcripción reversa utilizando una transcriptasa reversa de virus de leucemia murina (Molones, Promega), según protocolo descrito ${ }^{12}$.

Amplificación de región del genoma donde se producen mutaciones asociadas a resistencia. Se realizó una primera RPC para amplificar la zona de la proteína M2 donde se encuentran las mutaciones más frecuentes, utilizando los partidores recomendados por Saito y cols ${ }^{12}$. Luego se realizó una RPC anidada utilizando partidores correspondientes a la mutación 31 , descritos por el mismo autor. Los productos de RPC se visualizaron en un gel de electroforesis utilizando agarosa al $4 \%$.

Digestión con enzimas de restricción. Las hebras resultantes fueron sometidas a digestión por la enzima de restricción Sca I (Promega), según lo descrito en el protocolo utilizado como referencia $^{12}$. 
Electroforesis en gel de agarosa. Los fragmentos obtenidos post digestión fueron sometidos a electroforesis en gel de agarosa en forma simultánea a los fragmentos sin digestión. Se utilizó un marcador de peso molecular estándar (50-bp ADN).

\section{Resultados}

Se procesaron 33 muestras: 20 aisladas el año 2001 y 13 durante el año 2002. De ellas, 18 $(55 \%)$ pertenecían a pacientes de sexo femenino. Las edades fluctuaron entre 4 meses y 74 años (mediana $=37$ años).

Se obtuvo información en 22 de los 33 pacientes $(67 \%)$ : en 10 casos mediante revisión de la ficha clínica y 12 fueron contactados telefónicamente. De estos 22 pacientes, $16(72 \%)$ tenían menos de 15 años y 11 (68\%) el antecedente de bronquitis obstructiva recurrente. De los 6 adultos, 4 tenían sobre 65 años de edad. De los 22 pacientes con información conocida, 16 (72\%) tenían indicación formal de vacunación; sin embargo, no la habían recibido. Ninguno de los pacientes recibió terapia antiviral. Un paciente (un adulto de 72 años) fue hospitalizado por neumonía por FLU A y requerir oxígenoterapia.

De las 33 muestras procesadas, 31 presentaron la banda de $150 \mathrm{bp}$ en el gel de electroforesis tras realizar la RPC anidada. Esta banda corresponde a la amplificación de la zona 31 de la proteína M2 (Figura 3). En las 2 muestras restantes no se obtuvo banda al repetir la RPC anidada. Un control negativo (agua) fue incluido para cada uno de los análisis.

Al realizar la digestión con la enzima de restricción específica, las 31 muestras sometidas a digestión mostraron una banda de 120 bp en el gel de electroforesis (Figura 4).

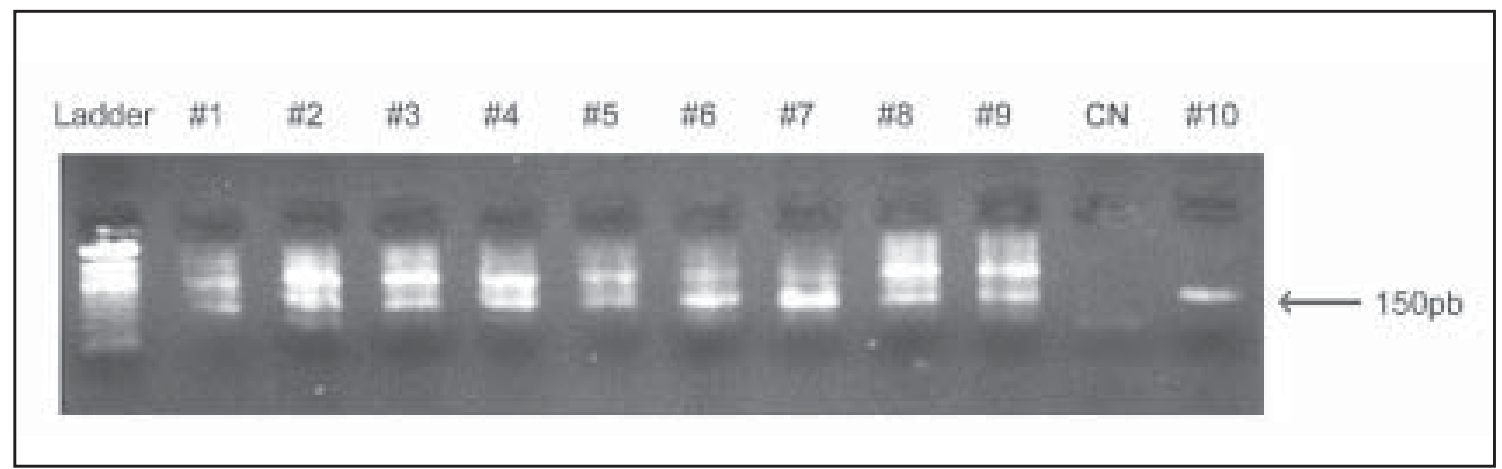

Figura 3. Amplificación segmento de 150 pb (zona 31 de proteína M2).

Ladder: marcador de talla molecular estándar de 50-pb ADN; \#1-10: muestras de pacientes; CN: control negativo (agua).

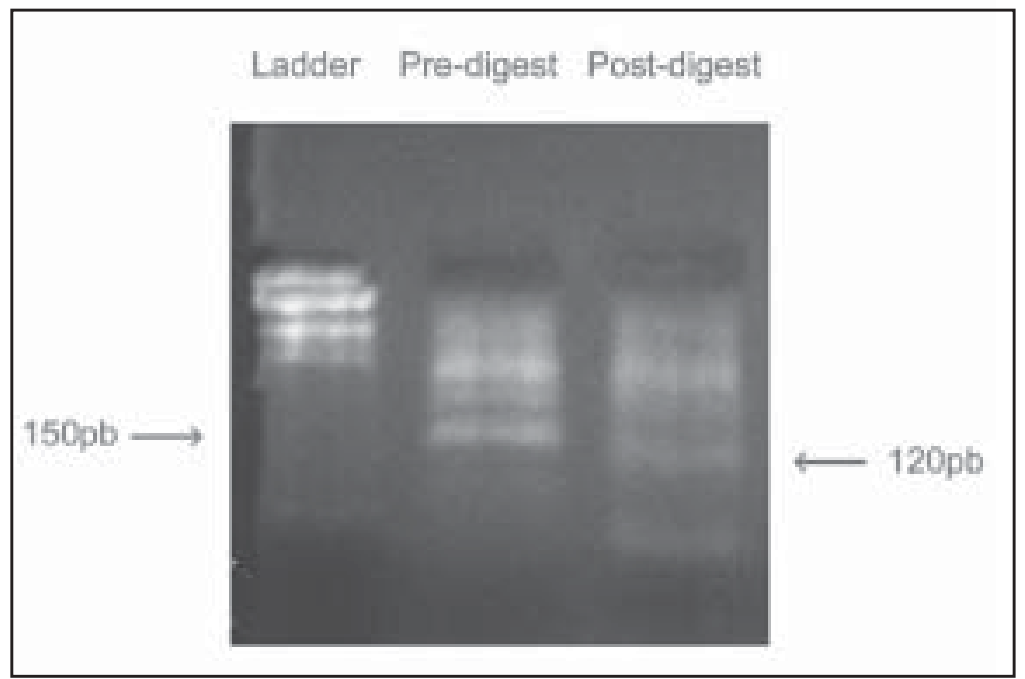

Figura 4. Electroforesis pre y post digestión con ScaI. Ladder: marcador de talla molecular estándar de 50-pb ADN; Pre digestión: producto del RPC anidado pre-digestión de 150 pb. Post digestión: producto del RPC anidado post-digestión con enzima de restricción Sca-I de 120pb. 


\section{Discusión}

Dada la gran complejidad del estudio de susceptibilidad de los virus a antivirales por metodología tradicional, como es el cultivo viral en presencia del antiviral, se hace importante contar con técnicas alternativas. Este estudio demuestra la utilidad de técnicas de biología molecular para estos efectos y su aplicabilidad en nuestro medio. En nuestro conocimiento, ésta es la primera experiencia nacional con esta metodología. Con ella, no se encontraron cepas de virus FLU A con la mutación 31, la más frecuentemente asociada con resistencia a amantadina. Estos resultados apoyan la hipótesis que en nuestra comunidad la resistencia del virus FLU a amantadina es muy baja o inexistente. Este hallazgo concuerda con algunas series que reportan una muy baja prevalencia de resistencia ${ }^{10,14}$ pero difiere de otras series que encuentran resistencia en 10 a $30 \%$ de las cepas ${ }^{6,9,11-13}$.

Estas diferencias pueden atribuirse a algunas características de la población estudiada, tales como edad, grado de institucionalización ${ }^{1,10}$ y frecuencia de uso de amantadina ${ }^{13}$. En este estudio no se incluyeron pacientes institucionalizados, mientras que las series con alta prevalencia de resistencia se han realizado en pacientes que residían en hogares de ancianos donde el hacinamiento es un factor de riesgo crucial para la adquisición de resistencia ${ }^{1,10}$. Si bien no existen datos respecto al uso de amantadina en nuestro medio, éste parece ser en general bajo y fundamentalmente es indicada en adultos. Nuestra población estuvo constituida principalmente por niños y ninguno de los pacientes en que pudo consultarse el antecedente había utilizado el antiviral.

Esta experiencia adolece de varias limitaciones; una de ellas es haber estudiado sólo una de las mutaciones conocidas, la más frecuente (responsable de más de $80 \%$ de las cepas resistentes). Por ello, no se puede inferir con certeza la ausencia de resistencia a amantadina en esta población, pero sí la hace muy poco probable. Por otro lado, el pequeño tamaño de la muestra estudiada y la ausencia de cepas provenientes de pacientes que hubieran recibido antes el antiviral, impide extrapolar conclusiones a toda la población. Lamentablemente no se pudo contar con un control positivo, esto es, una cepa de virus influenza resistente a amantadina, lo que habría dado mayor seguridad a los hallazgos negativos.

En conclusión, la técnica utilizada para el estudio de resistencia de virus FLU a amantadina podría ser de alta utilidad en nuestro país ante un mayor uso del medicamento o en circunstancias con alto riesgo de aparición de cepas resistentes, como brotes de FLU en asilos de ancianos u otros pacientes institucionalizados. Eventualmente, la metodología puede ser utilizada como mecanismo de vigilancia de resistencia antiviral.

Finalmente, sería de interés realizar estudios comparativos que evalúen el impacto del uso de amantadina en la adquisición de resistencia en nuestro medio.

\section{Resumen}

Amantadina es un fármaco eficaz para el tratamiento y prevención de influenza A. Su mecanismo de acción es inhibir la proteína M2. Su uso por períodos prolongados puede generar resistencia, la cual ocurre por mutaciones en el gen que codifica para la proteína M2. La mutación más frecuentemente encontrada se ubica en la posición 31. El uso de técnicas de biología molecular permite detectar estas mutaciones. Los objetivos fueron determinar la existencia de resistencia a amantadina en cepas de virus influenza A aisladas entre los años 2001 y 2002 en un laboratorio de virología en Santiago de Chile, y validar un nuevo método de biología molecular para reconocer cepas resistentes. Para ello se utilizó metodología de RPC y análisis de tamaño de fragmentos de restricción. En 31 cepas procesadas no se observó la presencia de cambios en la posición 31. Estos hallazgos sugieren que la resistencia a amantadina es muy baja o está ausente en nuestro medio. Esto podría explicarse por un limitado uso de este fármaco en esta población. El método descrito puede servir de base para un monitoreo prospectivo de resistencia, que pueda ser de utilidad al médico clínico.

\section{Bibliografía}

1.- Stiver G. The treatment of influenza with antiviral drugs. Can Med Assoc J 2003; 168: 49-56.

2.- Uyeki T. Influenza diagnosis and treatment in children: a review of studies on clinically useful tests and antiviral treatment for influenza. Pediatr Infect Dis J 2003; 22: 164-77.

3.- American Academy of Pediatrics. Influenza. En Pickering LK, ed. Red Book 2003 Report of the Committee on Infectious Diseases. $26^{\text {th }}$ ed. Elk Grove Village, Il: American Academy of Pediatrics; 2003: 382.

4.- Holsinger L, Nichani D, Pinto L, Lamb R. Influenza A virus M2 ion channel protein: a structure-function analysis. J Virol 1994; 68: 1551-63.

5.- Montalto N, Gum K, Ashley J. Updated treatment for influenza A and B. Am Fam Physician 2000; 62: $2467-$ 76.

6.- Kandel R, Hartshorn K L. Prophylaxis and treatment of influenza virus infection. BioDrugs 2001; 15: 303 23. 
7.- Jefferson T O, Demicheli V, Deeks J J, Rivetti D. Amantadine and rimantidine for preventing and treating influenza A in adults. Cochrane Database Syst Rev 2002; 3: CD001169

8.- Tooley P. Unprepared for an influenza pandemic. Lancet 2001; 358: 670-1.

9.- Houck P, Hemphill M, Lacroix S, Hirsh D, Cox N. Amantadine-resistant influenza A in nursing homes. Identification of a resistant virus prior to drug use. Arch Intern Med 1995; 155: 533-7.

10.- Tooley P. Drug resistance and influenza pandemics. Lancet 2002; 360: 1703-4.

11.- Stilianakis N, Perelson A, Hayden F. Drug resistance and influenza pandemics. Lancet 2002; 359: 1862-1.

12.- Saito R, Oshitani H, Masuda H, Suzuki H. Detection of amantadine-resistant influenza A virus strains in nursing homes by RPC-restriction fragment length polymorphism analysis with nasopharyngeal swabs. J Clin Microbiol 2002; 40: 84-8.

13.- Bridges CB, Harper S A, Fukuda K, Uyeki T M, Cox N J, Singleton J A. Prevention and control of influenza. Recommendations of the Advisory Committee on Immunization Practices (ACIP). MMWR Recomm Rep 2003; 52 (RR8): 1-34.

14.- Suzuki H, Saito R, Oshitani H. Excess amantadine use and resistant viruses. Lancet 2001; 358: 1910.

15.- Masuda H, Suzuki H, Oshitani H, Saito R, Kawasaki S, Nishikawa M, et al. Incidence of amantadine-resistant influenza A viruses in sentinel surveillance sites and nursing homes in Niigata, Japan. Microbiol Immunol 2000; 44: 833-9.

16.- Iwahashi J, Tsuji K, Ishibashi T, Kajiwara J, Imamura $\mathrm{Y}$, Mori R. et al. Isolation of amantadine-resistant influenza A viruses (H3N2) from patient following administration of amantadine in Japan. J Clin Microbiol 2001; 39: 1652-3.

17.- Shiraishi K, Mitamura K, Sakai-Tagawa Y, Goto H, Sugaya N, Kawaoka Y. High frequency of resistant viruses harboring different mutations in amantadinetreated children with influenza. J Infect Dis 2003; 188: 57-61.

18.- Klimov A, Rocha E, Hayden F, Shult P, Roumillat F, Cox N. Prolonged shedding of amantadine-resistant influenza A viruses by immunodeficient patients: detection by polymerase chain reaction restriction analysis. J Infect Dis 1995; 172: 1352-5.

19.- Ferrés M. Manual de Exámenes de Laboratorio y Vacunas Especiales. Laboratorio de Infectología y Virología Molecular de la Pontificia Universidad Católica. Santiago, 2005.

Correspondencia a:

Nicole Le Corre Pérez

mlec@puc.cl 